\title{
Ultraschall 2020: On the Move
}

Nachdem bereits vor Jahren (siehe «Praxis»-Editorials anlässlich der jährlichen SGUM-Fortbildungskurse) die ersten Schritte zur Implementierung der Ultraschallausbildung während der präklinischen und klinischen Jahre des Medizinstudiums erwogen und auch erprobt wurden [1], hat nun ein neues, griffiges und flächendeckendes Ausbildungskonzept für die gesamte Schweiz im Kontext des neuen Schweizer Lernzielkatalogs «PROFILES» Fuss gefasst. Ziel ist das Erlernen einfacher sonografischer Untersuchungen bereits während des medizinischen Grundstudiums. Dank der Initiative der sog. «Amikalen Runde», bestehend aus Vertretern der SGUM sowie aller Schweizer Universitäten, liegt nun ein Konsenspapier vor, das kürzlich in der SAEZ publiziert wurde [2]. Darin wird besonders das Konzept des «Blended Learning» hervorgehoben, das neben dem klassischen Tutorial unter Einbezug erprobter US-Tutoren und Tutorinnen das Erlernen sonografischer Grundbegriffe, v.a. auch aus dem Bereich Physik und sonografische Basis-Technologie, erlaubt [3, 4].

Definition und Anwendungsmöglichkeiten dieses Konzepts werden in der vorliegenden «Praxis»-Ausgabe illustriert. In diesem Zusammenhang ist auch die unter Leitung von Roman Hari (Berner Institut für Hausarztmedizin) initiierte «Signature»-Studie zu erwähnen, die das «Outcome» des «Blended Learning»-Konzepts mit den traditionellen, bisher von den Fakultäten angebotenen Ultraschallkursen für Studierende der Medizin vergleicht.

\section{Ausgangslage}

Das ubiquitäre Vorhandensein von Ultraschallgeräten in medizinischen Notfallstationen und Praxen hat dazu geführt, dass Ultraschalluntersuchungen auch ohne entsprechende Basisausbildung durchgeführt werden. Dieser Umstand hat zu einem oberflächlichen Einsatz des Ultraschalls und damit zu dessen Abwertung geführt. Die BasisUltraschallausbildung muss daher zwingend von der Weiterbildungsperiode in die universitäre Ausbildungsphase verschoben werden. Die Zeichen der Zeit sind nun auch von den Universitäten erkannt worden, und die vorliegenden Empfehlungen zielen auf eine sinnvolle Integration der Sonografie in die ärztliche Aus- und Weiterbildung ab. Entsprechend besteht vonseiten der Studierenden ein grosses Interesse, sich die Grundkenntnisse in diagnostischer Sonografie anzueignen.

Der «Amikalen Runde» ist es nun gelungen, den Schulterschluss zwischen der SGUM als für sämtliche Belange der diagnostischen Sonografie zuständige Organisation und universitären Institutionen zu schaffen, um auch der chronischen Überbuchung der bisher ausschliesslich optional angebotenen Sonografie-Basiskurse Abhilfe zu schaffen. Es geht dabei v.a. um das Erlernen der sonografi- schen Grundkonzepte unter Einschluss der physikalischen und technischen Grundlagen sowie um Fragen der sinnvollen Implementierung der Sonografie in klinische Entscheidungsprozesse. Weiter sollten die Studierenden am Ende dieser Ausbildung fähig sein, einfache Ultraschallanwendungen praktisch durchzuführen [4].

Es geht dabei v.a. um das symptomzentrierte Erlernen spezifischer Fragestellungen unter Einschluss der 4 VIP (Very Important Pathologies): 1. Cholezystitis/Cholelithiasis, 2. Aortenaneurysma, 3. Harnwegsobstruktion, 4. tiefe Venenthrombose.

Damit sollten die angehenden Ärztinnen und Ärzte für den Einsatz dieses hilfreichen diagnostischen Tools im klinischen Alltag gewappnet sein.

Im vorliegenden Heft finden sich dazu auch interessante Fortbildungsartikel, die sich einerseits an versierte Ultraschallanwender wenden, andererseits aber auch «Ultraschallnovizen» einen Einblick in das breite Spektrum der klinischen Anwendungsmöglichkeiten des diagnostischen Ultraschalls ermöglichen.

\section{Fazit}

Zusammenfassend erscheint es daher wichtig, angehende Ärztinnen und Ärzte möglichst früh in die diagnostische Sonografie einzuführen, damit die jungen Kolleginnen und Kollegen bereits am Anfang ihrer Assistentenzeit dieses Triage-Tool auch praktisch anwenden können, v.a. im Rahmen des «Point-of-Care»-Konzepts [5].

\section{Bibliografie}

1. Tuma J, Schwarzenbach HR: Ultraschall als Bestandteil des Studentenunterrichts. Praxis 2016; 105: 677

2. Hari R, Meienberg A, Braun L: Ultraschallausbildung für Studierende in der Schweiz: Ein Consensus von Universitäten und der SGUM. Schweiz Ärzteztg 2020; 101: n251-253.

3. Arroyo-Morales M, Cantarero-Villanueva I, Fernández-Lao C, Guirao-Piñeyro M, Castro-Martín E, Díaz-Rodríguez L: A blended learning approach to palpation and ultrasound imaging skills through supplementation of traditional classroom teaching with an e-learning package. Man Ther 2012; $17: 474-478$

4. Lewiss RE, Hoffmann B, Beaulieu Y, Phelan MB: Point-of-care ultrasound education: The increasing role of simulation and multimedia resources. J Ultrasound Med 2014; 33: 27-32.

5. Moore, CL,Copel CA: Point-of-care ultrasonography. N Engl J Med 2011;364:749-757.

Dr. med. Hans-Rudolf Schwarzenbach

Via Pocobelli 16

6815 Melide

hans-rudolf.schwarzenbach@hin.ch 